\title{
Condition number of the BEM matrix arising from the Stokes equations in $2 \mathrm{D}$
}

\author{
W. Dijkstra*a, R.M.M. Mattheij* \\ *Department of Mathematics and Computing Science, Eindhoven University of Technology, \\ P.O. Box 513, 5600 MB Eindhoven, The Netherlands \\ ${ }^{a}$ w.dijkstra@tue.nl
}

\begin{abstract}
We study the condition number of the system matrices that appear in the Boundary Element Method when solving the Stokes equations at a two-dimensional domain. At the boundary of the domain we impose Dirichlet conditions or mixed conditions. We show that for certain critical boundary contours the underlying boundary integral equation is not solvable. As a consequence, the condition number of the system matrix of the discrete equations is infinitely large. Hence, for these critical contours the Stokes equations cannot be solved with the Boundary Element Method. To overcome this problem the domain can be rescaled. Several numerical examples are provided to illustrate the solvability problems at the critical contours.
\end{abstract}

\section{Introduction}

Nowadays the Boundary Element Method (BEM) is widely used by mathematicians and engineers in a variety of fields, such as heat and fluid flow, structural mechanics, acoustics, etc. Despite the succes of the BEM, little attention has been paid to a fundamental issue as solvability of the equations that appear in the BEM. In general the BEM amounts to solving a system of linear algebraic equations. The solvability of this system depends to a large extent on the condition number of the system matrix. Hence knowledge about this condition number is essential when investigating the solvability of the system of equations.

It was shown that the condition number of the BEM-matrix for the Laplace equation in $2 D$ with Dirichlet conditions may become infinitely large when the logarithmic capacity of the domain of interest is equal to one $[2,3,4,6,9]$. This is also true for the Laplace equation with mixed boundary conditions [10]. The condition number of the BEM matrix for the Helmholtz equation may also become infinitely large $[1,15,16]$. This happens at a countable set of critical wave numbers. In all these cases where the condition number gets infinitely large, solving the linear system is very difficult.

In general, to investigate the condition number of the BEM-matrix, the underlying boundary integral equations need to be studied. These equations and the operators that appear in them have been the topic of several papers. Again it is noted that the boundary integral equation for the Laplace equation with Dirichlet conditions is not uniquely solvable when the logarithmic capacity of the domain is equal to one $[13,14,19,22]$. A similar result is true for the biharmonic equation with Dirichlet conditions [5, 8, 12].

In this paper we concentrate on the Stokes equations supplemented with Dirichlet or mixed boundary conditions. We investigate the solvability of the boundary integral formulation of these equations. For this goal we study the single-layer operator that appears in the formulation and see under what conditions it admits zero eigenvalues. In Section 2 we briefly describe the boundary 
integral equation for the Stokes problem, introducing the single and double layer operator. For the case of a circular domain the eigenvalues and eigenfunctions of the single-layer operator can be calculated analytically. These results are presented in Section 3. In Section 4 we show that the single-layer operator admits zero eigenvalues under certain conditions. As a consequence the boundary integral equation for Stokes flow with Dirichlet conditions is not always solvable. The same holds for the boundary integral equation for Stokes flow with mixed boundary conditions. This is proved in Section 5. Section 6 illustrates the findings by computing the condition number of the BEM-matrix for several examples.

\section{Boundary integral equations for 2D Stokes flow}

Let $\Omega$ be a two-dimensional simply-connected domain $\Omega$ with smooth boundary contour $\Gamma$. The Stokes equations for a flow in $\Omega$ read

$$
\begin{aligned}
\nabla^{2} \boldsymbol{v}-\nabla p & =0, \\
\nabla \cdot \boldsymbol{v} & =0,
\end{aligned}
$$

where $\boldsymbol{v}$ is the velocity field of the fluid and $p$ its pressure. Let $\Gamma$ be divided into a part $\Gamma_{1}$ at which the velocity $\boldsymbol{v}$ is prescribed, and a part $\Gamma_{2}$ at which the pressure $p$ is prescribed, $\Gamma=\Gamma_{1} \bigcup \Gamma_{2}$. The Stokes equations are subject to the boundary conditions

$$
\begin{aligned}
& \boldsymbol{v}=\tilde{\boldsymbol{v}}, \boldsymbol{x} \in \Gamma_{1}, \\
& p=\tilde{p}, \boldsymbol{x} \in \Gamma_{2} .
\end{aligned}
$$

Either $\Gamma_{1}$ or $\Gamma_{2}$ can be empty, leading to a pure Neuman or Dirichlet problem respectively. The Stokes equations in differential form can be transformed to the boundary integral equation (for details about the derivation we refer to $[17,19,20]$ )

$$
\frac{1}{2} v_{i}(\boldsymbol{x})+\int_{\Gamma} q_{i j}(\boldsymbol{x}, \boldsymbol{y}) v_{j}(\boldsymbol{y}) d \Gamma_{y}=\int_{\Gamma} u_{i j}(\boldsymbol{x}, \boldsymbol{y}) b_{j}(\boldsymbol{y}) d \Gamma_{y}, \boldsymbol{x} \in \Gamma, i=1,2 .
$$

Here a repeated index means summation over all possible values of that index. The vector function $\boldsymbol{b}$ is the normal stress at the fluid boundary,

$$
\boldsymbol{b}:=\mathcal{T}(p, \boldsymbol{v}) \boldsymbol{n},
$$

with $\boldsymbol{n}$ the outward unit normal at the boundary and the stress tensor $\mathcal{T}$ defined as

$$
\mathcal{T}_{i j}(p, \boldsymbol{v}):=-p \delta_{i j}+\left(\frac{\partial v_{i}}{\partial x_{j}}+\frac{\partial v_{j}}{\partial x_{i}}\right)
$$

Hence the boundary integral formulation involves two variables, the velocity $\boldsymbol{v}$ and the normal stress $\boldsymbol{b}$. At each point of the boundary either $\boldsymbol{v}$ or $\boldsymbol{b}$ is prescribed,

$$
\begin{aligned}
& \boldsymbol{v}=\tilde{\boldsymbol{v}}, \boldsymbol{x} \in \Gamma_{1}, \\
& \boldsymbol{b}=-\tilde{p} \boldsymbol{n}, \boldsymbol{x} \in \Gamma_{2} .
\end{aligned}
$$

The kernels $u_{i j}$ and $q_{i j}$ in the integral equations are defined as

$$
\begin{aligned}
q_{i j}(\boldsymbol{x}, \boldsymbol{y}) & =\frac{1}{\pi} \frac{\left(x_{i}-y_{i}\right)\left(x_{j}-y_{j}\right)\left(x_{k}-y_{k}\right) n_{k}}{\|\boldsymbol{x}-\boldsymbol{y}\|^{4}}, \\
u_{i j}(\boldsymbol{x}, \boldsymbol{y}) & =\frac{1}{4 \pi}\left\{-\delta_{i j} \log \|\boldsymbol{x}-\boldsymbol{y}\|+\frac{\left(x_{i}-y_{i}\right)\left(x_{j}-y_{j}\right)}{\|\boldsymbol{x}-\boldsymbol{y}\|^{2}}\right\} .
\end{aligned}
$$

We introduce boundary integral operators,

$$
\begin{aligned}
(\mathcal{G} \varphi)_{i}(\boldsymbol{x}) & =\int_{\Gamma} u_{i j}(\boldsymbol{x}, \boldsymbol{y}) \varphi_{j}(\boldsymbol{y}) d \Gamma_{y} \\
(\mathcal{H} \psi)_{i}(\boldsymbol{x}) & =\int_{\Gamma} q_{i j}(\boldsymbol{x}, \boldsymbol{y}) \psi_{j}(\boldsymbol{y}) d \Gamma_{y}
\end{aligned}
$$


which enables us to write (3) as

$$
\left(\frac{1}{2} \mathcal{I}+\mathcal{H}\right) \boldsymbol{v}=\mathcal{G} \boldsymbol{b}
$$

The operators $\mathcal{G}$ and $\mathcal{H}$ are often called the single and double layer operator for the Stokes equations. For the Dirichlet problem the velocity $\boldsymbol{v}$ at the boundary is given $\left(\Gamma_{2}=\emptyset\right)$ and we aim to reconstruct the normal stress $\boldsymbol{b}$ at the boundary by solving

$$
\mathcal{G} \boldsymbol{b}=\boldsymbol{f}(\tilde{\boldsymbol{v}}):=\left(\frac{1}{2} \mathcal{I}+\mathcal{H}\right) \tilde{\boldsymbol{v}}
$$

To solve this equation we need to invert the operator $\mathcal{G}$. This can only be done when all eigenvalues of $\mathcal{G}$ are unequal to zero. In this paper we investigate under what conditions $\mathcal{G}$ admits a zero eigenvalue.

For the mixed problem the velocity at $\Gamma_{1}$ is prescribed and the normal stress at $\Gamma_{2}$ is prescribed. By rearranging known and unknown terms (see Section 5) the system (9) can be written as

$$
\mathcal{A x}=\boldsymbol{g} .
$$

Again, to solve this equation we need to invert the operator $\mathcal{A}$. This can only be done when all eigenvalues of $\mathcal{A}$ are unequal to zero. We will show that zero eigenvalues occur under the same conditions as for the Dirichlet problem.

\section{Eigensystem of the single layer operator $\mathcal{G}$ at a circular domain}

In this section we compute eigenfunctions and eigenvalues of the operator $\mathcal{G}$ for a circular boundary $\Gamma$. Here we make use of the fact that the first term in the kernel $u_{i j}$ is equal (apart from a constant) to the fundamental solution for the Laplace operator in $2 D$. Hence the first term from the single-layer operator $\mathcal{G}$ for the Stokes equations is related to the single-layer operator $\left(\mathcal{K}^{s}\right)$ for the Laplace equation by

$$
\left.\frac{1}{4 \pi} \int_{\Gamma}(-\log \|\boldsymbol{x}-\boldsymbol{y}\|) b(\boldsymbol{y})\right) d \Gamma_{y}=\frac{1}{2}\left(\mathcal{K}^{s} b\right)(\boldsymbol{x}) .
$$

For $\Gamma$ a circle with radius $R$, the single-layer potential $\mathcal{K}^{s}$ admits the following eigensystem $([9])$,

$$
\begin{aligned}
\mathcal{K}^{s} \cos (k t) & =\frac{R}{2 k} \cos (k t), \\
\mathcal{K}^{s} \sin (k t) & =\frac{R}{2 k} \sin (k t), \\
\mathcal{K}^{s} 1 & =-R \log R .
\end{aligned}
$$

We introduce the following polar coordinates,

$$
\boldsymbol{x}=R[\cos t, \sin t], \quad \boldsymbol{y}=R[\cos s, \sin s] .
$$

Then the distance squared between $\boldsymbol{x}$ and $\boldsymbol{y}$ is computed as $\|\boldsymbol{x}-\boldsymbol{y}\|^{2}=2 R^{2}(1-\cos (t-s))$ and we find

$$
\begin{aligned}
& u_{11}(t, s)=\frac{1}{8 \pi}\left\{-\delta_{i j} \log \left[2 R^{2}(1-\cos (t-s))\right]+\frac{(\cos t-\cos s)^{2}}{1-\cos (t-s)}\right\} \\
& u_{12}(t, s)=\frac{1}{8 \pi} \frac{(\cos t-\cos s)(\sin t-\sin s)}{1-\cos (t-s)} \\
& u_{21}(t, s)=\frac{1}{8 \pi} \frac{(\sin t-\sin s)(\cos t-\cos s)}{1-\cos (t-s)}=u_{12}(t, s) \\
& u_{22}(t, s)=\frac{1}{8 \pi}\left\{-\delta_{i j} \log \left[2 R^{2}(1-\cos (t-s))\right]+\frac{(\sin t-\sin s)^{2}}{1-\cos (t-s)}\right\} .
\end{aligned}
$$




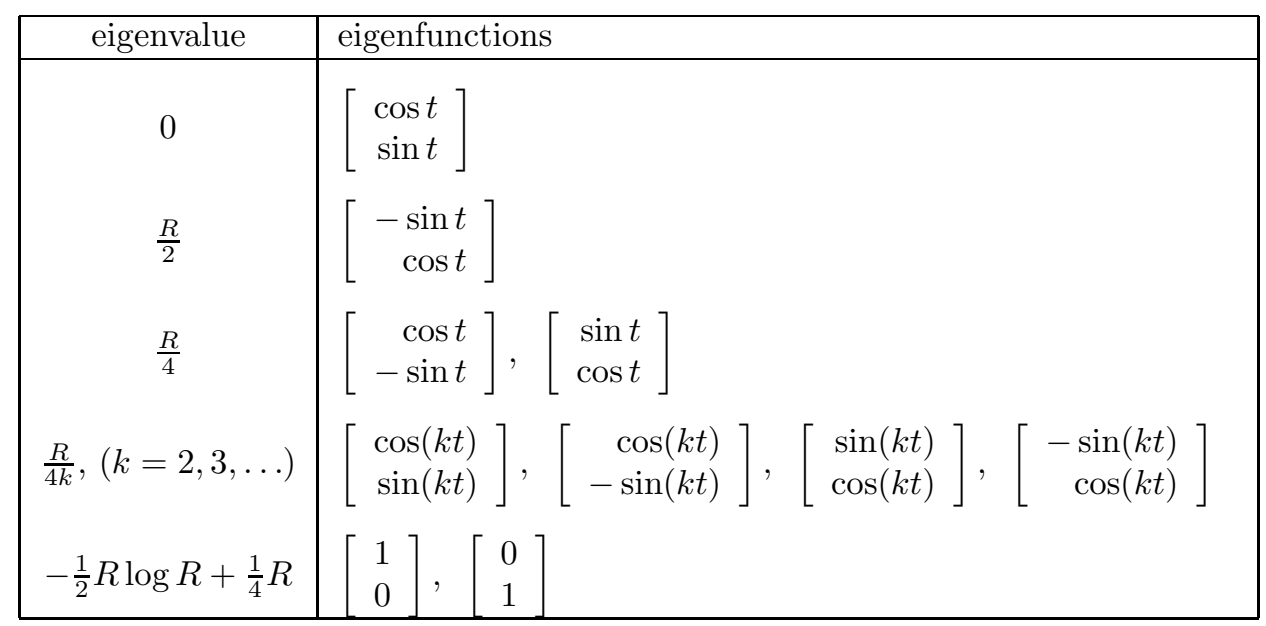

Table 1: Eigenvalues and eigenfunctions of $\mathcal{G}$.

Using the integral kernels in polar coordinates given in (15) it is straightforward to compute the eigenvalues and eigenvectors of the operator $\mathcal{G}$. The results are given in Table 1.

We observe that the function $\boldsymbol{b}(\boldsymbol{x})=\boldsymbol{n}=[\cos t, \sin t]^{T}$ is an eigenfunction with eigenvalue zero. This can be explained as follows: when we apply a stress in the direction of the normal at each point of the boundary, each stress having the same magnitude, the net contribution will be equal to zero. This phenomenon does not restrict to the circle, but applies to any shape of the boundary, as will be proven in Section 4.

For the case $R=R^{*}:=\exp (1 / 2)$, we see that there is another zero eigenvalue which has an eigenspace with dimension two. The eigenfunctions correspond to a uniform stress distribution; at each point at the boundary an equal stress is applied in the same direction. This particular stress distribution will cause a translation of the body. The circular boundary contour with $R=R^{*}$ is often called a critical contour (or $\Gamma$-contour [14]). The radius or scale $R^{*}$ is often called the critical scale or degenerate scale. Equation (10) at the critical contour cannot be solved as the operator $\mathcal{G}$ admits a zero eigenvalue.

The situation in which the boundary integral equation is not solvable for a critical size of the circle also occurs for the Dirichlet Laplace equation in $2 D[2,3,4,6,9]$. It is shown that the single-layer operator for the unit circle admits a zero eigenvalue, and consequently it cannot be inverted. Hence, the same phenomenon appears for both the Laplace and Stokes equations. The critical scale however is not the same for the Laplace and Stokes equations.

\section{Invertibility of single layer operator on general domain}

In this section we study the solvability of the boundary integral equation (10) on a smooth closed boundary contour $\Gamma$. We search for eigenfunctions $\boldsymbol{b}$ of the boundary integral operator $\mathcal{G}$ with zero eigenvalue, hence $\mathcal{G} \boldsymbol{b}=0$. If such eigenfunctions exist the integral equation (10) is not uniquely solvable.

Theorem 1 For any smooth boundary $\Gamma$ the outward unit normal $\boldsymbol{n}$ is an eigenfunction of the boundary integral operator $\mathcal{G}$ with eigenvalue zero. 
Proof. The $i$-th component of $\mathcal{G} \boldsymbol{n}$ equals

$$
\begin{aligned}
(\mathcal{G} \boldsymbol{n})_{i} & =\int_{\Gamma} u_{i j}(\boldsymbol{x}, \boldsymbol{y}) n_{j}(\boldsymbol{y}) d \Gamma_{y} \\
& =\frac{1}{4 \pi} \int_{\Gamma}\left[\delta_{i j} \log \frac{1}{\|\boldsymbol{x}-\boldsymbol{y}\|}+\frac{\left(x_{i}-y_{i}\right)\left(x_{j}-y_{j}\right)}{\|\boldsymbol{x}-\boldsymbol{y}\|^{2}}\right] n_{j}(\boldsymbol{y}) d \Gamma_{y} \\
& =\frac{1}{4 \pi} \int_{\Omega} \frac{\partial}{\partial x_{j}}\left[\delta_{i j} \log \frac{1}{\|\boldsymbol{x}-\boldsymbol{y}\|}+\frac{\left(x_{i}-y_{i}\right)\left(x_{j}-y_{j}\right)}{\|\boldsymbol{x}-\boldsymbol{y}\|^{2}}\right] d \Omega_{y} \\
& =-\int_{\Omega} \frac{\partial}{\partial x_{j}} u_{j}^{i} d \Omega_{y}=-\int_{\Omega} \operatorname{div} \boldsymbol{u}^{i} d \Omega=0 .
\end{aligned}
$$

Here $\boldsymbol{u}^{i}$ is the flow velocity due to a Stokeslet [23]. This velocity satisfies the incompressibility condition $\operatorname{div} \boldsymbol{u}^{i}=0$.

In Section 3 we already saw that for a circular boundary the normal vector $\boldsymbol{n}$ is an eigenfunction of $\mathcal{G}$ with eigenvalue zero. The current theorem generalizes this result to arbitrary smooth closed boundary contours. In the sequel of this section we assume that the solutions of the Dirichlet problem (10) are sought in a function space that excludes the unit normal. Hence the eigenfunctions $\boldsymbol{b}$ of $\mathcal{G}$ we are looking for cannot be equal to $\boldsymbol{n}$.

We now show that for each boundary $\Gamma$ there exist (atmost) two critical scalings of the boundary such that the operator $\mathcal{G}$ in the Dirichlet problem (10) is not invertible. This phenomenon has been observed and proven by [11] and we will partly present their analysis here.

Theorem 2 For all given functions $\boldsymbol{f}$ and constant vectors $\boldsymbol{d}$ the system of equations

$$
\left\{\begin{aligned}
\mathcal{G} b+\boldsymbol{c} & =\boldsymbol{f} \\
\int_{\Gamma} \boldsymbol{b} d \Gamma & =\boldsymbol{d}
\end{aligned}\right.
$$

has a unique solution pair $(\boldsymbol{b}, \boldsymbol{c})$, where $\boldsymbol{b}$ is a function and $\boldsymbol{c}$ a constant vector.

Proof. The main idea is to show that the operator that maps the pair $(\boldsymbol{b}, \boldsymbol{c})$ to the left-hand side of (17) is an isomorphism. For details we refer to [11].

We proceed by introducing the two unit vectors $\boldsymbol{e}_{1}=[1,0]^{T}$ and $\boldsymbol{e}_{2}=[0,1]^{T}$. Theorem 2 quarantees that two pairs $\left(\boldsymbol{b}^{1}, \boldsymbol{c}^{1}\right)$ and $\left(\boldsymbol{b}^{2}, \boldsymbol{c}^{2}\right)$ exist that are the unique solutions to the two systems

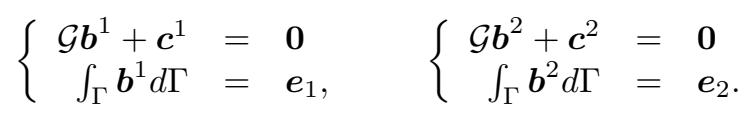

We define the matrix $\mathbf{C}_{\Gamma}$ as $\mathbf{C}_{\Gamma}:=\left[\boldsymbol{c}^{1} \mid \boldsymbol{c}^{2}\right]$.

Theorem 3 If $\operatorname{det}\left(\mathbf{C}_{\Gamma}\right)=0$, then the operator $\mathcal{G}$ is not invertible.

Proof. Suppose that $\operatorname{det}\left(\mathbf{C}_{\Gamma}\right)=0$, then the columns $\boldsymbol{c}^{1}$ and $\boldsymbol{c}^{2}$ are dependent, say $\boldsymbol{c}^{1}=\alpha \boldsymbol{c}^{2}$ for some $\alpha \in \mathbb{R}, \alpha \neq 0$. In that case

$$
\mathbf{0}=\left(\mathcal{G} \boldsymbol{b}^{1}+\boldsymbol{c}^{1}\right)-\alpha\left(\mathcal{G} \boldsymbol{b}^{2}+\boldsymbol{c}^{2}\right)=\mathcal{G}\left(\boldsymbol{b}^{1}-\alpha \boldsymbol{b}^{2}\right)+\alpha \boldsymbol{c}^{2}-\alpha \boldsymbol{c}^{2}=\mathcal{G}\left(\boldsymbol{b}^{1}-\alpha \boldsymbol{b}^{2}\right) .
$$

The function $\boldsymbol{b}^{1}-\alpha \boldsymbol{b}^{2}$ cannot be equal to zero, since this would require $\int_{\Gamma}\left(\boldsymbol{b}^{1}-\alpha \boldsymbol{b}^{2}\right) d \Gamma$ to be equal to zero, while we have

$$
\int_{\Gamma}\left(\boldsymbol{b}^{1}-\alpha \boldsymbol{b}^{2}\right) d \Gamma=\boldsymbol{e}_{1}-\alpha \boldsymbol{e}_{2} \neq \mathbf{0} .
$$

So $\boldsymbol{b}^{1}-\alpha \boldsymbol{b}^{2}$ is an eigenfunction of $\mathcal{G}$ with zero eigenvalue. (Note that this eigenfunction cannot be equal to the normal $\boldsymbol{n}$ at the boundary, since $\boldsymbol{n}$ satisfies $\int_{\Gamma} \boldsymbol{n} d \Gamma=0$.)

Corollary 1 There are (atmost) two critical scalings of the domain $\Gamma$ for which the operator $\mathcal{G}$ is not invertible. 
Proof. We rescale the domain $\Gamma$ by a factor $a$, i.e. $\Gamma \rightarrow a \Gamma$. With the definition of the operator $\mathcal{G}$ it can be shown that

$$
\mathcal{G} \boldsymbol{b} \rightarrow \mathcal{G}^{a} \boldsymbol{b}:=-\frac{1}{4 \pi} \int_{\Gamma} a \log a \boldsymbol{b} d \Gamma+a \mathcal{G} \boldsymbol{b} .
$$

Then the two systems in (18) change into

$$
\left\{\begin{aligned}
a \mathcal{G} \boldsymbol{b}^{j}+\boldsymbol{c}^{j}-\frac{1}{4 \pi} \int_{\Gamma} a \log a \boldsymbol{b}^{j} d \Gamma & =\mathbf{0} \\
a \int_{\Gamma} \boldsymbol{b}^{j} d \Gamma & =\boldsymbol{e}_{j}, j=1,2 .
\end{aligned}\right.
$$

Define $\boldsymbol{b}_{a}^{j}:=a \boldsymbol{b}^{j}$ for $j=1,2$, then we obtain

$$
\left\{\begin{aligned}
\mathcal{G} \boldsymbol{b}_{a}^{j}+\boldsymbol{c}^{j}-\frac{1}{4 \pi} \int_{\Gamma} \log a \boldsymbol{b}_{a}^{j} d \Gamma & =\mathbf{0} \\
\int_{\Gamma} \boldsymbol{b}_{a}^{j} d \Gamma & =\boldsymbol{e}_{j}, j=1,2
\end{aligned}\right.
$$

Substituting the second equation into the first equation, we get

$$
\left\{\begin{aligned}
\mathcal{G} \boldsymbol{b}_{a}^{j}+\boldsymbol{c}^{j}-\frac{1}{4 \pi} \log a \boldsymbol{e}_{j} & =\mathbf{0} \\
\int_{\Gamma} \boldsymbol{b}_{a}^{j} d \Gamma & =\boldsymbol{e}_{j}, j=1,2
\end{aligned}\right.
$$

These systems have the same form as the original systems in (18), with $\boldsymbol{c}^{j} \rightarrow \boldsymbol{c}^{j}-\frac{1}{4 \pi} \log a \boldsymbol{e}_{j}$ for $j=1,2$. Define the new matrix $\mathbf{C}_{a \Gamma}$ by

$$
\mathbf{C}_{a \Gamma}:=\mathbf{C}_{\Gamma}-\frac{1}{4 \pi} \log a \mathbf{I}_{2},
$$

then $\mathcal{G}^{a}$ is not invertible when $\operatorname{det}\left(\mathbf{C}_{a \Gamma}\right)=0$. Hence when $\frac{1}{4 \pi} \log a$ is an eigenvalue of $\mathbf{C}_{\Gamma}$ the operator $\mathcal{G}^{a}$ is not invertible. This implies that, when $\mathbf{C}_{\Gamma}$ has two distinct eigenvalues, there are two critical sizes $a^{*}$ for which $\mathcal{G}^{a}$ is not invertible. If $\mathbf{C}_{\Gamma}$ has one eigenvalue with double multiplicity these critical scalings coincide.

This result shows that the solvability of the boundary integral equation for the Dirichlet Stokes problem depends on the scale of the domain. There exist (atmost) two critical scales for which the equations are not solvable. The solvability problem is an artifact of the boundary integral formulation; the Stokes equations inn differential form are well-posed. For the Laplace equation with Dirichlet conditions a similar phenomenon is observed [13, 14, 19, 22]. In its differential form the problem is well-posed, while the corresponding boundary integral equation is not solvable at critical boundary contours.

REMARK: The boundary integral equations for the Stokes flow in 2D are similar to the equations for plane elasticity. Hence the BIE for the latter equations suffer from the same solvability problems as the Stokes equations. A proof of this phenomenon for plane elasticity has been presented in $[25]$ and is similar to the proof sketched above.

\section{Invertibility of operator on general domain with mixed boundary conditions}

In the previous section we showed that the boundary integral operator $\mathcal{G}$ for the Dirichlet Stokes equations is not invertible for all boundaries. In this section we show that this phenomenon extends to the Stokes equations with mixed boundary conditions.

The starting point is again the BIE for the Stokes equations,

$$
\frac{1}{2} \boldsymbol{v}+\mathcal{H} \boldsymbol{v}=\mathcal{G} \boldsymbol{b}, \text { at } \Gamma .
$$

Suppose that the boundary $\Gamma$ is split into two parts, $\Gamma=\Gamma_{1} \bigcup \Gamma_{2}$. At $\Gamma_{1}$ we prescribe the velocity $\boldsymbol{v}^{1}$ while the normal stress $\boldsymbol{b}^{1}$ is unknown. At $\Gamma_{2}$ we prescribe the normal stress $\boldsymbol{b}^{2}$ while the 
velocity $\boldsymbol{v}^{2}$ is unknown. The boundary integral operators $\mathcal{G}$ and $\mathcal{H}$ are split accordingly,

$$
\begin{aligned}
{[\mathcal{G} \boldsymbol{b}]_{i} } & =\int_{\Gamma} u_{i j} b_{j} d \Gamma=\int_{\Gamma_{1}} u_{i j} b_{j}^{1} d \Gamma+\int_{\Gamma_{2}} u_{i j} b_{j}^{2} d \Gamma=:\left[\mathcal{G}^{1} \boldsymbol{b}^{1}\right]_{i}+\left[\mathcal{G}^{2} \boldsymbol{b}^{2}\right]_{i} \\
{[\mathcal{H} \boldsymbol{v}]_{i} } & =\int_{\Gamma} q_{i j} v_{j} d \Gamma=\int_{\Gamma_{1}} q_{i j} v_{j}^{1} d \Gamma+\int_{\Gamma_{2}} q_{i j} v_{j}^{2} d \Gamma=:\left[\mathcal{H}^{1} \boldsymbol{v}^{1}\right]_{i}+\left[\mathcal{H}^{2} \boldsymbol{v}^{2}\right]_{i}
\end{aligned}
$$

With these notations the BIE is written in the following way,

$$
\frac{1}{2} \boldsymbol{v}^{k}+\mathcal{H}^{1} \boldsymbol{v}^{1}+\mathcal{H}^{2} \boldsymbol{v}^{2}=\mathcal{G}^{1} \boldsymbol{b}^{1}+\mathcal{G}^{2} \boldsymbol{b}^{2}, \text { at } \Gamma_{k}, k=1,2 .
$$

We arrange the terms in such a way that all unknowns are at the left-hand side and all knowns are at the right-hand side,

$$
\begin{aligned}
\mathcal{H}^{2} \boldsymbol{v}^{2}-\mathcal{G}^{1} \boldsymbol{b}^{1} & =\mathcal{G}^{2} \boldsymbol{b}^{2}-\mathcal{H}^{1} \boldsymbol{v}^{1}-\frac{1}{2} \boldsymbol{v}^{1}, \text { at } \Gamma_{1}, \\
\frac{1}{2} \boldsymbol{v}^{2}+\mathcal{H}^{2} \boldsymbol{v}^{2}-\mathcal{G}^{1} \boldsymbol{b}^{1} & =\mathcal{G}^{2} \boldsymbol{b}^{2}-\mathcal{H}^{1} \boldsymbol{v}^{1}, \text { at } \Gamma_{2} .
\end{aligned}
$$

Now we can define an operator $\mathcal{A}$ that assigns to the pair $\left(\boldsymbol{b}^{1}, \boldsymbol{v}^{2}\right)$ the two functions at the left-hand side of (29),

$$
\left[\begin{array}{c}
\boldsymbol{b}^{1} \\
\boldsymbol{v}^{2}
\end{array}\right] \stackrel{\mathcal{A}}{\rightarrow}\left[\begin{array}{r}
\mathcal{H}^{2} \boldsymbol{v}^{2}-\mathcal{G}^{1} \boldsymbol{b}^{1} \\
\frac{1}{2} \boldsymbol{v}^{2}+\mathcal{H}^{2} \boldsymbol{v}^{2}-\mathcal{G}^{1} \boldsymbol{b}^{1}
\end{array}\right]
$$

To study the invertibility of this operator we need to study the homogeneous version of the equations in (29),

$$
\begin{aligned}
\mathcal{H}^{2} \boldsymbol{v}^{2}-\mathcal{G}^{1} \boldsymbol{b}^{1} & =0, \text { at } \Gamma_{1}, \\
\frac{1}{2} \boldsymbol{v}^{2}+\mathcal{H}^{2} \boldsymbol{v}^{2}-\mathcal{G}^{1} \boldsymbol{b}^{1} & =0, \text { at } \Gamma_{2} .
\end{aligned}
$$

Theorem 4 There are (atmost) two scalings of $\Gamma$ such that the homogeneous equations (31) have a non-trivial solution, i.e. $\mathcal{A}$ is not invertible.

Proof. From the Dirichlet problem we know that there are (atmost) two scalings of $\Gamma$ for which $\mathcal{G}$ is not invertible. So there are $a_{I}$ and $a_{I I} \in \mathbb{R}$ and vector functions $\boldsymbol{q}_{I}$ and $\boldsymbol{q}_{I I}$ such that

$$
\mathcal{G} \boldsymbol{q}_{k}=\mathbf{0}, \text { at } a_{k} \Gamma, k=I, I I .
$$

The scalings $a_{k}$ and functions $q_{k}$ may coincide but this does not affect the proof of the theorem. Denote the nullspace of $\mathcal{G}$ by $N(\mathcal{G})$. The normal vector $\boldsymbol{n}$ is always in $N(\mathcal{G})$. If $\Gamma$ is a critical contour then also $\boldsymbol{q}_{I}$ and $\boldsymbol{q}_{I I}$ are in $N(\mathcal{G})$. Assume that $\Gamma$ is such a critical contour. Let $\boldsymbol{q} \in N(\mathcal{G})$, that is $\boldsymbol{q}=\alpha_{1} \boldsymbol{q}_{I}+\alpha_{2} \boldsymbol{q}_{I I}+\alpha_{3} \boldsymbol{n}$, for some $\alpha_{1}, \alpha_{2}$ and $\alpha_{3}$ in $\mathbb{R}$, and $\mathcal{G} \boldsymbol{q}=\mathbf{0}$. Consider the homogeneous equations (31). We will show that there is a non-trivial pair $\left(\boldsymbol{b}^{1}, \boldsymbol{v}^{2}\right)$ that satisfies these equations. We choose $\boldsymbol{v}^{2}=\mathbf{0}$ and $\boldsymbol{b}^{1}=\boldsymbol{q}^{1}+\boldsymbol{h}^{1}$. Here $\boldsymbol{q}^{i}$ is the restriction of $\boldsymbol{q}$ to $\Gamma_{i}, i=1,2$, and $\boldsymbol{h}^{1}$ is still unknown. Substituting these functions in the left-hand sides of the homogeneous equations yields for both equations

$$
-\mathcal{G}^{1} \boldsymbol{b}^{1}=-\mathcal{G}^{1} \boldsymbol{q}^{1}-\mathcal{G}^{1} \boldsymbol{h}^{1}=+\mathcal{G}^{2} \boldsymbol{q}^{2}-\mathcal{G}^{1} \boldsymbol{h}^{1},
$$

where we made use of $\mathcal{G} \boldsymbol{q}=\mathcal{G}^{1} \boldsymbol{q}^{1}+\mathcal{G}^{2} \boldsymbol{q}^{2}=0$. If we can find a function $\boldsymbol{h}^{1}$ such that $\mathcal{G}^{1} \boldsymbol{h}^{1}=\mathcal{G}^{2} \boldsymbol{q}^{2}$, then the left-hand sides of the homogeneous equations yield zero and we have found a non-trivial solution. Our task is then to prove that a solution $\boldsymbol{h}^{1}$ of

$$
\mathcal{G}^{1} \boldsymbol{h}^{1}=\mathcal{G}^{2} \boldsymbol{q}^{2}
$$

exists. A function $\boldsymbol{b}$ is in $N(\mathcal{G})^{\perp}$ if the inner product of $\boldsymbol{q}$ and $\boldsymbol{b}$ is equal to zero, i.e.

$$
\int_{\Gamma} q_{i}(\boldsymbol{x}) b_{i}(\boldsymbol{x}) d \Gamma_{x}=0
$$


First we note that the right-hand side of $(34)$ is contained in $N(\mathcal{G})^{\perp}$, since

$$
\begin{aligned}
\int_{G} q_{i}\left[\mathcal{G}^{2} \boldsymbol{q}^{2}\right]_{i} d \Gamma_{x} & =\int_{\Gamma} q_{i}(\boldsymbol{x}) \int_{\Gamma_{2}} u_{i j}(\boldsymbol{x}, \boldsymbol{y}) q_{j}^{2}(\boldsymbol{y}) d \Gamma_{y} d \Gamma_{x} \\
& =\int_{\Gamma_{2}} q_{j}^{2}(\boldsymbol{y}) \int_{\Gamma} u_{i j}(\boldsymbol{x}, \boldsymbol{y}) q_{i}(\boldsymbol{x}) d \Gamma_{x} d \Gamma_{y} \\
& =\int_{\Gamma_{2}} q_{j}^{2}(\boldsymbol{y})[\mathcal{G} \boldsymbol{q}]_{j} d \Gamma_{y}=0 .
\end{aligned}
$$

So we may generalize our task: prove that a solution $\boldsymbol{h}^{1}$ of

$$
\mathcal{G}^{1} \boldsymbol{h}^{1}=\phi
$$

exists, for any $\phi \in N(\mathcal{G})^{\perp}$. If so, then $\phi=\mathcal{G}^{2} \boldsymbol{q}^{2}$ completes the proof. It is known that the operator $\mathcal{G}$ is a Fredholm operator with index zero [7]. Hence the Fredholm alternative can be applied [18, p. 37]. This states that for the homogeneous equation two mutually exclusive possibilities exist:

1. $\mathcal{G} \boldsymbol{h}=\mathbf{0}$ only has the trivial solution;

2. $\mathcal{G} \boldsymbol{h}=\mathbf{0}$ has exactly $p$ linearly independent solutions.

We are in the second siuation, as the nullspace of $\mathcal{G}$ is non-empty and is spanned by $\boldsymbol{n}, \boldsymbol{q}_{I}$ and $\boldsymbol{q}_{I I}$. The Fredholm alternative then states that $\mathcal{G} \boldsymbol{h}=\boldsymbol{\phi}$ is solvable if and only if $\boldsymbol{\phi}$ is perpendicular to the $p$ independent solutions of $\mathcal{G}^{*} \boldsymbol{f}=\mathbf{0}$, where $\mathcal{G}^{*}$ denotes the adjoined operator of $\mathcal{G}$. However, the single layer operator for the Stokes flow is self-adjoined, so $\mathcal{G}^{*}=\mathcal{G}$. Consequently the solvability condition says that $\boldsymbol{\phi}$ has to be perpendicular to the solutions of $\mathcal{G} \boldsymbol{h}=\mathbf{0}$. This is the case since we defined $\boldsymbol{\phi} \in N(\mathcal{G})^{\perp}$. Consequently there exists an $\boldsymbol{h}$ with $\mathcal{G} \boldsymbol{h}=\boldsymbol{\phi}$, for $\boldsymbol{\phi} \in N(\mathcal{G})^{\perp}$. We split this $\boldsymbol{h}$ into two parts,

$$
\boldsymbol{h}= \begin{cases}\boldsymbol{h}^{1}, & \text { at } \Gamma_{1}, \\ \boldsymbol{h}^{2}, & \text { at } \Gamma_{2} .\end{cases}
$$

Recall that we search for $\boldsymbol{h}^{1}$ such that $\mathcal{G}^{1} \boldsymbol{h}^{1}=\boldsymbol{\phi}$. We add $\mathcal{G}^{2} \boldsymbol{h}^{2}$ to both sides of this equation, obtaining

$$
\mathcal{G}^{1} \boldsymbol{h}^{1}+\mathcal{G}^{2} \boldsymbol{h}^{2}=\boldsymbol{\phi}+\mathcal{G}^{2} \boldsymbol{h}^{2}
$$

or shorter

$$
\mathcal{G} \boldsymbol{h}=\boldsymbol{\phi}+\mathcal{G}^{2} \boldsymbol{h}^{2} .
$$

The right-hand side of this equation is in $N(\mathcal{G})^{\perp}$, since $\phi \in N(\mathcal{G})^{\perp}$ and

$$
\begin{aligned}
\int_{G} q_{i}\left[\mathcal{G}^{2} \boldsymbol{h}^{2}\right]_{i} d \Gamma_{x} & =\int_{\Gamma} q_{i}(\boldsymbol{x}) \int_{\Gamma_{2}} u_{i j}(\boldsymbol{x}, \boldsymbol{y}) h_{j}^{2}(\boldsymbol{y}) d \Gamma_{y} d \Gamma_{x} \\
& =\int_{\Gamma_{2}} h_{j}^{2}(\boldsymbol{y}) \int_{\Gamma} u_{i j}(\boldsymbol{x}, \boldsymbol{y}) q_{i}(\boldsymbol{x}) d \Gamma_{x} d \Gamma_{y} \\
& =\int_{\Gamma_{2}} h_{j}^{2}(\boldsymbol{y})[\mathcal{G} \boldsymbol{q}]_{j} d \Gamma_{y}=0
\end{aligned}
$$

so $\mathcal{G}^{2} \boldsymbol{h}^{2}$ is also in $N(\mathcal{G})^{\perp}$. Recall that $\mathcal{G} \boldsymbol{h}=\boldsymbol{\phi}+\mathcal{G}^{2} \boldsymbol{h}^{2}$ is solvable if the right-hand side is in $N(\mathcal{G})^{\perp}$, so there exists an $\boldsymbol{h}$ satisfying (40). Then we may construct $\boldsymbol{h}^{1}$ by simply restricting $\boldsymbol{h}$ to $\Gamma_{1}$, i.e. $\boldsymbol{h}^{1}=\left.\boldsymbol{h}\right|_{\Gamma_{1}}$.

This results shows that also the boundary integral equation for the Stokes equations with mixed boundary conditions is not always solvable. This happens for the same critical boundary contours as for the Stokes equations with Dirichlet boundary conditions. Hence the mixed problem inherits the solvability problems from the Dirichlet problem. The division of the boundary into a Dirichlet and a Neuman part does not play a role in this.

Note that the Laplace equation exhibits the same behavior. The boundary integral equation for the Laplace equation with mixed boundary conditions also inherits the solvability problems from the boundary integral equation for the Dirichlet case [10]. 


\section{Numerical examples}

To solve the boundary integral equation (9), the boundary $\Gamma$ is discretised into a set of linear elements. We choose a set of $N$ nodes $\boldsymbol{x}^{p}(p=1, \ldots, N)$ at the boundary where two consecutive nodes $\boldsymbol{x}^{k 1}$ and $\boldsymbol{x}^{k 2}$ are connected by a straight line segment $\Gamma_{k}$. A point $\boldsymbol{y}$ at the element $\Gamma_{k}$ is described by $\boldsymbol{y}=\phi_{1}(s) \boldsymbol{x}^{k 1}+\phi_{2}(s) \boldsymbol{x}^{k 2}$, where $\phi_{1}$ and $\phi_{2}$ are linear shape functions, given by $\phi_{1}(s)=\frac{1}{2}(1-s)$ and $\phi_{2}(s)=\frac{1}{2}(1+s),-1 \leq s \leq 1$. At each element $\Gamma_{k}$ the velocity $\boldsymbol{v}$ and normal stress $\boldsymbol{b}$ are approximated with the same shape functions $\phi_{i}$, according to

$$
\begin{aligned}
& \boldsymbol{v}=\phi_{1}(s) \boldsymbol{v}^{k 1}+\phi_{2}(s) \boldsymbol{v}^{k 2}, \\
& \boldsymbol{b}=\phi_{1}(s) \boldsymbol{b}^{k 1}+\phi_{2}(s) \boldsymbol{b}^{k 2} .
\end{aligned}
$$

The vectors $\boldsymbol{v}^{p}$ and $\boldsymbol{b}^{p}$ are the velocity and normal stress at the node $\boldsymbol{x}^{p}$. We substitute $\boldsymbol{x}=\boldsymbol{x}^{p}$ at the boundary integral equation (3) and replace the integral over the boundary $\Gamma$ by a sum of integrals over the boundary elements $\Gamma_{k}$. This results in

$$
\begin{aligned}
\frac{1}{2} v_{i}\left(\boldsymbol{x}^{p}\right) & +\sum_{k=1}^{N} \int_{\Gamma_{k}} q_{i j}\left(\boldsymbol{x}^{p}, \boldsymbol{y}(s)\right)\left(\phi_{1}(s) v_{j}^{k 1}+\phi_{2}(s) v_{j}^{k 2}\right) d s|\boldsymbol{y}(s)| \\
& =\sum_{k=1}^{N} \int_{\Gamma_{k}} u_{i j}\left(\boldsymbol{x}^{p}, \boldsymbol{y}(s)\right)\left(\phi_{1}(s) b_{j}^{k 1}+\phi_{2}(s) b_{j}^{k 2}\right) d s|\boldsymbol{y}(s)|, i=1,2 .
\end{aligned}
$$

Varying $p$ from 1 to $N$, equation (43) yields $2 N$ equations for $4 N$ coefficients $v_{j}^{p}$ and $b_{j}^{p}$. However at each point at the boundary we either prescribe $\boldsymbol{v}$ or $\boldsymbol{b}$, thus reducing the number of coefficients by $2 N$. Hence we obtain a system of $2 N$ linear algebraic equations for $2 N$ unknown coefficients. We introduce vectors $\mathbf{v}$ and $\mathbf{b}$ of length $2 N$ containing the coefficients of $\boldsymbol{v}$ and $\boldsymbol{b}$ in the nodes. Then the system of equations can be written in short-hand notation as

$$
\left(\frac{1}{2} \mathbf{I}+\mathbf{H}\right) \mathbf{v}=\mathbf{G b} .
$$

To obtain the matrices $\mathbf{H}$ and $\mathbf{G}$ integrals of the form

$$
\int_{\Gamma_{k}} q_{i j}\left(\boldsymbol{x}^{p}, \boldsymbol{y}(s)\right) \phi_{r}(s) d s|\boldsymbol{y}(s)|, \quad \int_{\Gamma_{k}} u_{i j}\left(\boldsymbol{x}^{p}, \boldsymbol{y}(s)\right) \phi_{r}(s) d s|\boldsymbol{y}(s)|,
$$

have to be evaluated, respectively. These integrals can be approximated numerically by applying a Gaussian quadrature formula. When $\boldsymbol{x}^{p}$ is in the element $\Gamma_{k}$ over which integration is performed, the integrands will have a singularity in the integration domain. However, in the case of linear elements such as we use here, these integrals can be calculated analytically.

\subsection{Dirichlet boundary conditions}

For the Dirichlet problem, the coefficients of the velocity vector $\mathbf{v}$ are given, say $\mathbf{v}=\tilde{\mathbf{v}}$, and we need to solve

$$
\mathbf{G b}=\mathbf{f}(\tilde{\mathbf{v}}):=\left(\frac{1}{2} \mathbf{I}+\mathbf{H}\right) \tilde{\mathbf{v}}
$$

to find the unknown coefficients of the normal stress vector $\mathbf{b}$.

If the boundary integral operator $\mathcal{G}$ is not invertible then also its discrete counterpart, the matrix $\mathbf{G}$ is not invertible. To visualize this we compute the condition number of $\mathbf{G}$ : if the condition number is infinitely large, then the matrix is not invertible. As a consequence, (46) cannot be inverted to obtain $\mathbf{b}$.

In the following example we choose an ellipsoidal boundary contour $\Gamma$. We construct the matrix $\mathbf{G}$ and compute its condition number. Then we rescale the boundary $\Gamma$ by a factor $a$, i.e. $\Gamma \rightarrow a \Gamma$. Again we compute the condition number of the matrix G. We do this for several values of $a$. According to the theory in the previous section there are two critical values for $a$ for which the condition number of $\mathbf{G}$ goes to infinity. 
In Figure 1 we show the condition number as a function of the scale $a$. We do this for an ellipse with aspect ratio 0.4 (ellipse 1) and for an ellipse with aspect ratio 0.7 (ellipse 2). We observe that for both cases two critical scaling exist for which the condition number goes to infinity. Moreover, these critical values differ significantly for the two ellipses.

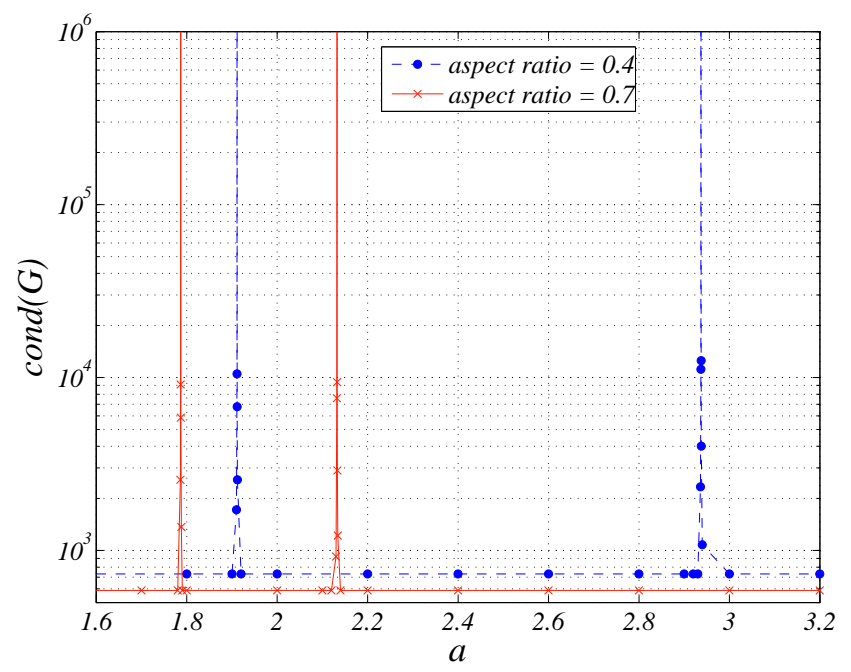

Figure 1: Condition number of $\mathbf{G}$ for an ellipsoidal domain with aspect ratios 0.4 and 0.7 as a function of scaling parameter a.

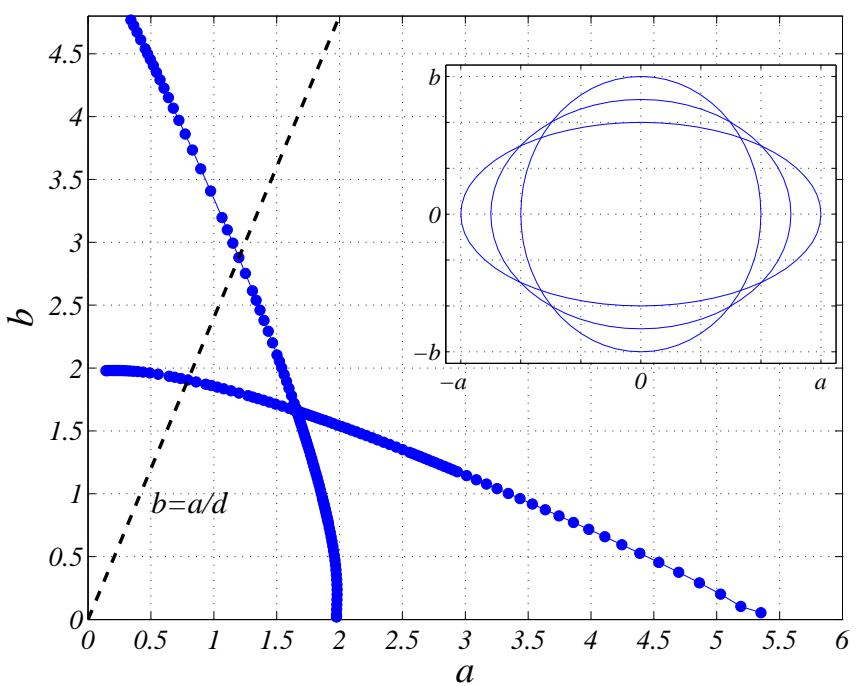

Figure 2: The critical sizes of an ellipse for which the condition number of $\mathbf{G}$ is very large.

Figure 2 visualises all ellipses for which the condition number of $\mathbf{G}$ is very large. At the horizontal axis we put the length $a$ of the horizontal semi-axis of the ellipse, at the vertical axis the length $b$ of the vertical semi-axis of the ellipse. We compute the condition number of $\mathbf{G}$ for several values of $a$ and $b$. We call the values of $a$ and $b$ for which the condition number goes to infinity the critical values and the corresponding ellipse the critical ellipse. At the critical values we plot a dot in the $(a, b)$-plane of Figure 2 . We see that the critical values lie on two curved lines, 
which are symmetric around the line $a=b$. The black dashed line with slope $1 / d$ represents all ellipses with aspect ratio $d:=a / b$. It can be concluded that for a fixed $d$, i.e. an ellipse with a fixed aspect ratio, two critical sizes exist. For a circle, where $d=1$, only one critical size exists. The values corresponding to this critical size are approximately $a=b=1.65$, which agrees with the eigenvalue of $\exp (1 / 2) \approx 1.649$ that we found in Section 3 .
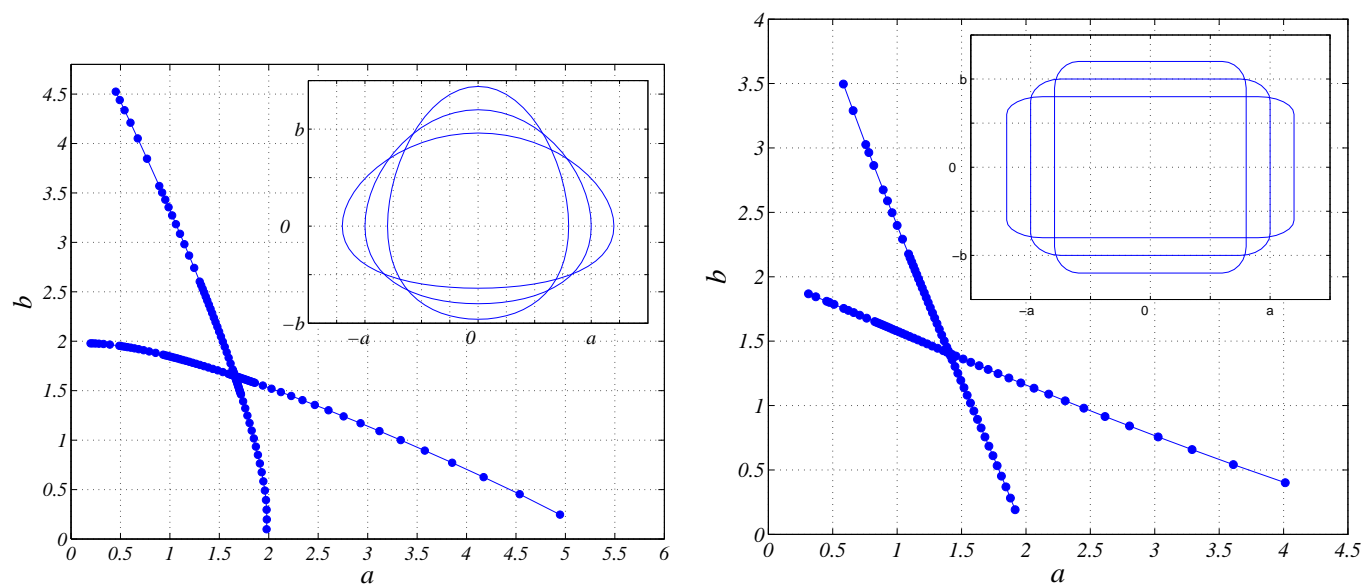

Figure 3: The critical sizes of two fluid domains.

In Figure 3 we have shown the critical sizes for two other boundary contours. For both cases the results look similar to those of the ellipse. Again there are always two critical sizes, except when the aspect ratio of the domain is equal to one.

\subsection{Mixed boundary conditions}

In the case of mixed boundary conditions we may rearrange terms in (44) and put all known coefficients at the right-hand side and all unknown coefficients at the left-hand side. Then we obtain a linear system of the form

$$
\mathrm{Ax}=\mathrm{g} .
$$

The matrix $\mathbf{A}$ consists of a block from the matrix $\mathbf{G}$ and a block from the matrix $\mathbf{H}$. We compute the condition number of the matrix $\mathbf{A}$ for the case of an ellipsoidal boundary contour. The ellipse is approximated by 16 linear elements. At the first eight elements we impose Dirichlet boundary conditions and at the last eight elements we impose Neuman boundary conditions. Then we rescale the boundary by a factor $a$ and again compute the condition number. Figure 4 shows the condition number of the matrix for an ellipse with aspect ratio 0.4 and an ellipse with aspect ratio 0.7 . We see that there exist two critical scalings for each ellipse. For these critical ellipses the condition number of $\mathbf{A}$ becomes infinitely large.

The critical sizes of the ellipse in the case of mixed conditions are close to the critical sizes for the case of Dirichlet conditions. In Figure 5 we show all critical sizes of the ellipse for the case of mixed conditions. One can observe that this plot is almost similar to the plot in Figure 2 for Dirichlet conditions. This illustrates the idea that the boundary integral equation for the mixed case inherits the solvability problem from the boundary integral equation for the Dirichlet case. Still some small differences can be distinguished in the critical scalings for the Dirichlet and mixed case. These differences are caused by errors in the numerical computations. When the number of boundary elements increases, the differences between the critical scalings descreases. 


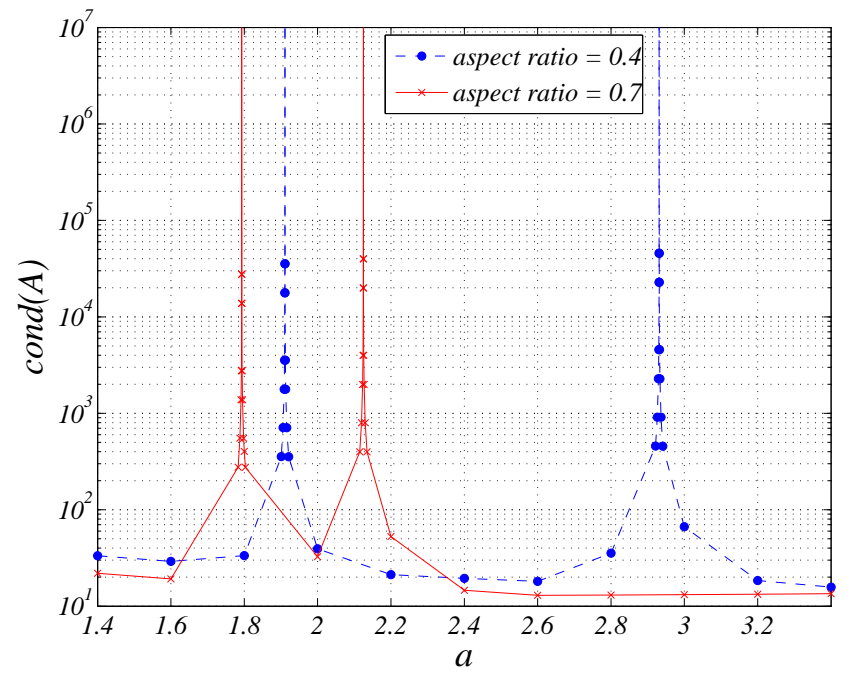

Figure 4: Condition number of $\mathbf{A}$ for an ellipsoidal domain with aspect ratios 0.4 and 0.7 as a function of scale $a$.

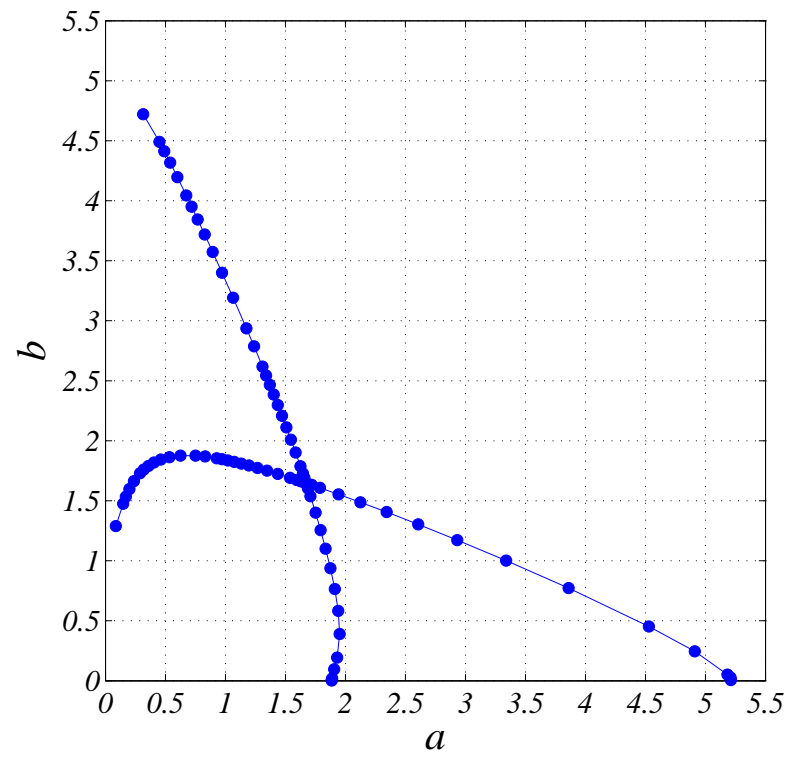

Figure 5: The critical sizes of an ellipse for which the condition number of $\mathbf{A}$ is very large.

\subsection{Deformation of viscous drop due to surface tension}

We now turn our attention to a time-dependent problem. We study a viscous drop of fluid of ellipsoidal shape that deforms to a circle due to surface tension. The evolution of the boundary of the drop is governed by the Stokes equations and can be solved using the Boundary Element Method [21, 24]. The velocity $\boldsymbol{v}$ of the boundary and the normal stress $\boldsymbol{b}$ at the boundary are related by the boundary integral equation (9). The normal stress $\boldsymbol{b}$ is proportional to the mean curvature $\kappa$ of the boundary,

$$
\boldsymbol{b}=-2 \gamma \kappa \boldsymbol{n},
$$


with $\gamma$ the surface tension and $\boldsymbol{n}$ the outward normal at the boundary. At time level $t=t_{1}$ we compute the velocity $\boldsymbol{v}$ at all discretisation nodes $\boldsymbol{x}$. Then we update the boundary of the drop with an Euler forward step,

$$
\boldsymbol{x} \rightarrow \boldsymbol{x}+\Delta t \boldsymbol{v}(\boldsymbol{x})
$$

obtaining a new boundary contour. For this new boundary we again compute the velocity, and perform another Euler forward step. In this way we can study the shape evolution of the boundary. In Figure 6 we see the evolution of the ellipse to a circle. We choose 40 points to discretise the boundary, the size of the time step is $\Delta t=0.375$ and we compute 10 time steps. The initial shape is an ellipse with aspect ratio 0.5 and the longest semi-axis has a length 1.2 .

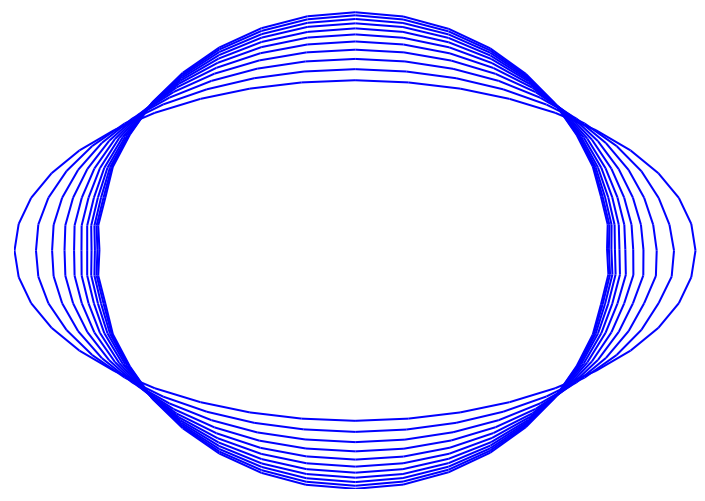

Figure 6: An ellipse deforming to a circle.

In the problem described above the normal stress is prescribed and the velocity has to be reconstructed with equation (10). For the inverse problem we try to reconstruct the normal stress given the boundary velocity, and therefore we need to invert the matrix $\mathbf{G}$ in each time step. We know that there are certain critical boundary contours for which the matrix $\mathbf{G}$ will not be invertible. In the problem of the deforming ellipse we go through a whole range of ellispes with different shapes, and we risk to encounter one or more of those critical contours.

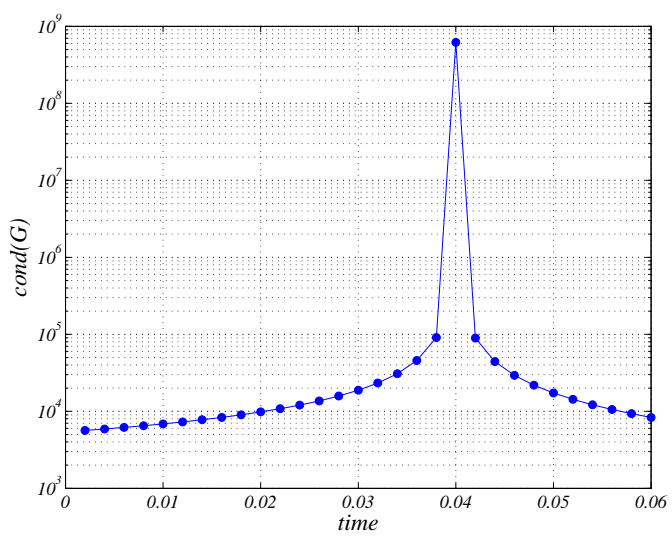

(a) Without scaling

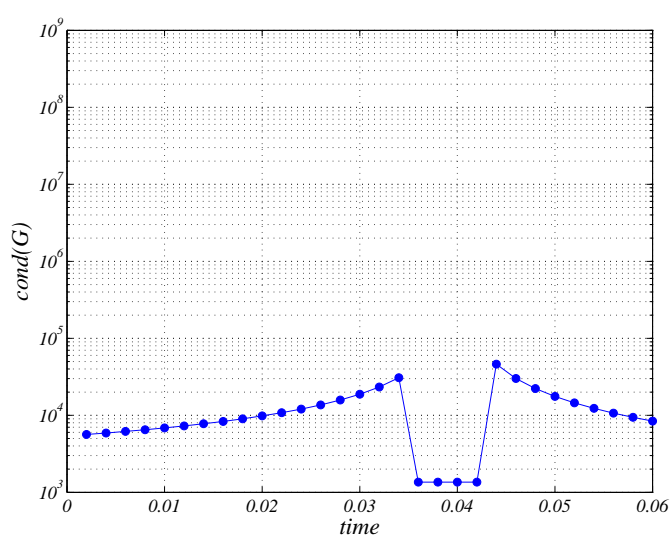

(b) With scaling close to critical size

Figure 7: The condition number of $\mathbf{G}$ at each time step.

In Figure 7(a) we show the condition number of the matrix $\mathbf{G}$ at each time step. An increase in the condition number indicates that the boundary contour under consideration is close to a 


\begin{tabular}{|c|rrc|}
\hline & without scaling & with scaling & difference \\
\hline area & 10.59729837 & 10.5972852 & $1.3150 e-05$ \\
length & 12.97535126 & 12.9751271 & $2.2412 e-04$ \\
$a$ & 2.759578290 & 2.75949155 & $8.6740 e-05$ \\
$b$ & 1.241866240 & 1.24190168 & $-3.5440 e-05$ \\
\hline
\end{tabular}

Table 2: Difference in the BEM with and without temporarily rescaling. Listed are the surface area, the length of the boundary and the length of the semi-axes at time $t=0.06$.

critical contour. We see that there is one time level in which such a critical contour is reached. In this case we choose 20 discretisation points, a time step size $\Delta t=0.002$ and 30 time steps.

There is a simple way to avoid a critical contour. When the size of the contour gets close to the critical contour, we temporarily scale the domain. In Figure 7(b) we show the outcome of this strategy. At each time step between $t=0.038$ and $t=0.046$ we scale the domain by a factor 1.02 . Then we solve the BEM problem in each of these time steps and the solutions are rescaled by a factor 1/1.02. As a consequence the condition number of the BEM-matrices in these time steps does not become very large. The scaling of the domain during some time steps does hardly affect the outcome of the test. In Table 2 we give the surface area, the length of the boundary contour and the sizes of the semi-axes at the final time step. Their values hardly change when we perform temporary scaling.

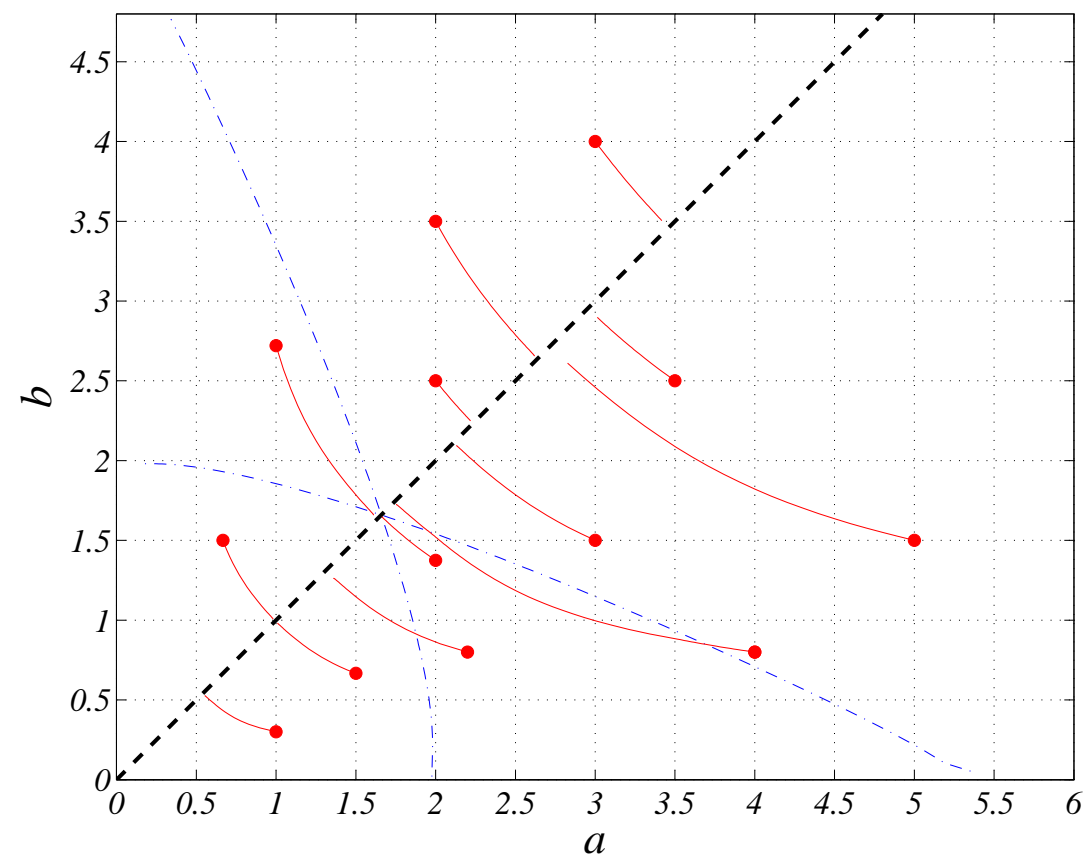

Figure 8: Each solid line represents the size of an ellipse as it deforms to a circle. The initial size, i.e. the lengths $a$ and $b$ of the semi-axes, is denoted with a large dot. The dashed-dotted lines represent the critical sizes. The thick black dashed line represents all circles.

Of course, for this strategy certain knowledge is needed about the critical contours. However, we do not need to know the exact critical contour. We only have to make sure we do not get too close to it. In the current test we only scaled at a restricted number of time steps. In general one 
could scale at every time step, thus excluding the possibility that a critical contour is encountered.

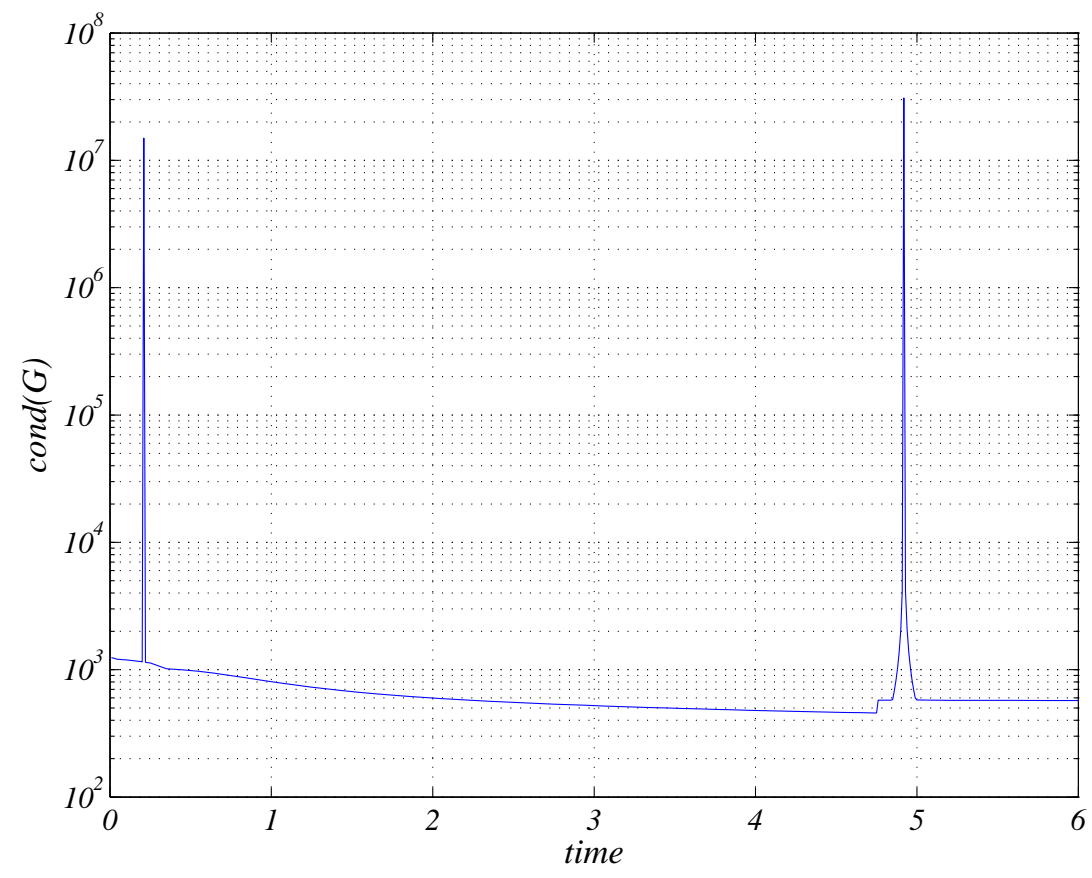

Figure 9: The condition number for the ellipse with initial values $a=4$ and $b=0.8$. During the deformation to a circle this particular ellipse gets a critical size twice.

The size of the initial ellipse strongly affects the condition number of the matrix at each time level as the ellipse deforms. In Figure 8 we show the size $(a$ and $b)$ of an ellipse as it is deforming. The size of the initial ellipse is shown with a large dot, whereas the sizes it takes as it deforms to a circle is denoted by the trajectory starting in the dot. All ellipses deform to a circle, which is seen from the fact that all trajectories converge to the straight thick dashed line where $a=b$.

The dashed-dotted lines represent all critical ellispes, c.f. Fifure 2. When the trajectory of an ellipse crosses one of the two dashed-dotted lines, it means that the ellipse at that time level is a critical ellipse. For this particular ellipse the condition number will be large. The trajectories of some ellipses never cross such a critical line. This means that they never get a critical size as they deform to a circle. For other ellipses there is one point along their trajectory where the ellipse is a critical ellipse. It is also possible that an ellipse becomes critical twice during the deformation. An example is the ellipse with initial size $a=4$ and $b=0.8$. The trajectory of this ellipse crosses the critical line twice. Figure 9 shows the condition number for this particular case. It can be seen that the condition number gets very large at the beginning and at the end of the time interval.

\section{Discussion}

The boundary integral equation for the Stokes equations with Dirichlet conditions at a twodimensional domain is not always uniquely solvable. The existence of a unique solution depends on the size of the domain. For each domain there is at least one size, or better one scaling, fow which the boundary integral does not have a unique solution. For most domains there are even two of such scalings. In order to find a unique solution the domain can be rescaled by a suitable factor.

Similar solvability problems appear in the boundary integral equation for the Laplace problem with Dirichlet conditions at a two-dimensional domain. In this case the critical size of the domain 
for which the equation is not uniquely solvable relates to the logarithmic capacity of the domain.

We have proven that, in analogy to the Laplace problem with mixed boundary conditions, also the Stokes problem with mixed boundary conditions does not always have a unique solution. This happens at the same critical domains as for the Dirichlet problem. Hence, the division of the boundary into a Dirichlet part and a Neuman part does not play a role in this.

By using a suitable rescaling of the domain the solvability problems can be avoided adequately. However, to choose the right scaling some knowledge about the critical scalings is useful.

\section{References}

[1] S. Amini and S.M. Kirkup. Solution of Helmholtz equation in the exterior domain by elementary boundary integral methods. J. Comput. Phys., 118:208-221, 1995.

[2] S. Christiansen. Condition number of matrices derived from two classes of integral equations. Mat. Meth. Appl. Sci, 3:364-392, 1981.

[3] S. Christiansen. On two methods for elimination of non-unique solutions of an integral equation with logarithmic kernel. Appl. Anal., 13:1-18, 1982.

[4] S. Christiansen and P.C. Hansen. The effective condition number applied to error analysis of certain boundary colocation methods. J. Comp. Appl. Math., 54:15-35, 1994.

[5] S. Christiansen and P. Hougaard. An investigation of a pair of integral equations for the biharmonic problem. J. Inst. Maths. Applics, 22:15-27, 1978.

[6] S. Christiansen and J. Saranen. The conditioning of some numerical methods for the first kind boundary integral equations. J. Comp. Appl. Math., 67:43-58, 1996.

[7] M. Costabel. Boundary integral operators on Lipschitz domains: elementary results. SIAM J. Math. Anal., 19(3):613-626, 1988.

[8] M. Costabel and M. Dauge. On invertibility of the biharmonic single-layer potential operator. Integr. Equ. Oper. Theory, 24:46-67, 1996.

[9] W. Dijkstra and R.M.M. Mattheij. The condition number of the BEM-matrix arising from Laplace's equation. Electron. J. Bound. Elem., 4(2):67-81, 2006.

[10] W. Dijkstra and R.M.M. Mattheij. A relation between the the logarithmic capacity and the condition number of the BEM-matrices. Comm. Numer. Methods Engrg., 1(1):0-1, 2007.

[11] V. Domínguez and F.J. Sayas. A BEM-FEM overlapping algorithm for the Stokes equation. Appl. Math. Comput., pages 691-710, 2006.

[12] B. Fuglede. On a direct method of integral equations for solving the biharmonic dirichlet problem. ZAMM, 61:449-459, 1981.

[13] G.C. Hsiao. On the stability of integral equations of the first kind with logarithmic kernels. Arch. Ration. Mech. Anal., 94:179-192, 1986.

[14] M.A. Jaswon and G. Symm. Integral Equation Methods in Potential Theory and Elastostatics. Academic Press, London, 1977.

[15] S.M. Kirkup. The influence of the weighting parameter on the improved boundary element solution of the exterior Helmholtz equation. Wave Motion, 93:93-101, 1992.

[16] R. Kress and W.T. Spassov. On the condition number of boundary integral operators for the exterior Dirichlet problem for the Helmholtz equation. Numer. Math., pages 77-95, 1983. 
[17] O.A. Ladyzhenskaya. The Mathematical Theory of Viscous Incompressible Flow. Gordon and Beach, New York-London, 1963.

[18] W. McLean and T. Tran. A preconditioning strategy for boundary element galerkin methods. Numer. Methods Partial Differential Eq., 13:283-301, 1997.

[19] H. Power and L.C. Wrobel. Boundary integral methods in fluid mechanics. Computational Mechanics Publications, Southampton, 1995.

[20] C. Pozrikidis. Boundary integral and singularity methods for linearized viscous flows. Cambridge University Press, Cambridge, 1992.

[21] A.R.M. Primo, L.C. Wrobel, and H. Power. An indirect boundary-element method for slow viscous flow in a bounded region containig air bubbles. J. Engrg. Math., 37:305-326, 2000.

[22] I.H. Sloan and A. Spence. The Galerkin method for integral equations of the first kind with logarithmic kernel: theory. IMA J. Numer. Anal., 8:105-122, 1988.

[23] G.A.L. van der Vorst. Modelling and numerical simulation of viscous sintering. PhD thesis, Eindhoven University of Technology, 1994.

[24] G.A.L. van der Vorst, R.M.M. Mattheij, and H.K. Kuiken. Boundary element solution for two-dimensional viscous sintering. J. Comput. Phys., 100:50-63, 1992.

[25] R. Vodicka and V. Mantic. On invertibility of elastic single-layer potential operator. $J$. Elasticity, 74:147-173, 2004. 